\title{
$\alpha$-Methylene- $\beta$-lactone probe for measuring live-cell reactions of small molecules
}

Lei Wang, ${ }^{\dagger}$ Louis P. Riel, ${ }^{\dagger}$ Bekim Bajrami, ${ }^{\ddagger}$ Bin Deng, ${ }^{\S}, \|$ Amy R. Howell, ${ }^{*}{ }^{\dagger}$ and Xudong Yao*,†,

†Department of Chemistry, University of Connecticut, Storrs, Connecticut 06269, USA.

‡Chemical Biology \& Proteomics, Biogen, Cambridge, Massachusetts 02142, USA.

§Department of Biology, University of Vermont, Burlington, Vermont 05405, USA.

॥Vermont Genetics Network Proteomics Facility, University of Vermont, Burlington, Vermont 05405, USA.

$\perp$ Institute for Systems Biology, University of Connecticut, Storrs, Connecticut 06269, USA.

KEYWORDS: $\alpha$-methylene- $\beta$-lactone, warhead, covalent probe, covalent inhibitor, covalent drug, chemical proteomics, peptide-centric competitive activity-based protein profiling, tagging triplication, cysteinome, Orlistat, glutathione S-transferase 
ABSTRACT: The novel use of the $\alpha$-methylene- $\beta$-lactone (MeLac) moiety as a warhead of multiple electrophilic sites is reported. In this study, we demonstrate that a MeLac-alkyne is a competent covalent probe and reacts with diverse proteins in live cells. Proteomics analysis of affinity-enriched samples identifies probe-reacted proteins, resolves their modified peptides/residues, and thus characterizes probe-protein reactions. Unique methods are developed to evaluate confidence in the identification of the reacted proteins and modified peptides. Tandem mass spectra of the peptides reveal that MeLac reacts with nucleophilic cysteine, serine, lysine, threonine, and tyrosine residues, through either Michael addition or acyl addition. A peptide-centric proteomics platform, using MeLac-alkyne as the measurement probe, successfully analyzes the Orlistat selectivity in live HT-29 cells. MeLac is a versatile warhead demonstrating enormous potential to expedite the development of covalent probes and inhibitors in interrogating protein (re)activity. MeLac-empowered platforms in chemical proteomics are widely adaptable for measuring the live-cell action of reactive molecules. 
There is a revival of interest in using covalent drugs in human healthcare. ${ }^{1}$ They have prolonged selective engagement with their targets and improved pharmacodynamic properties. Yet, these reactive drugs unavoidably react with off-target proteins, leading to toxicity and safety concerns. Therefore, the measurement of selectivity profiles of covalent drugs and inhibitors for their reactions with the human proteome, preferably in live cells under physiological conditions, is critical. Chemical proteomics analysis of drug-protein reactions expedites the development of new drugs by revealing selective covalent inhibitors early, identifying toxicity liabilities, and helping mitigate the risk of late-stage failures. ${ }^{2}$ This analysis often uses a boutique probe made by installing a reporting group on a drug molecule. However, making a drug-derived probe can be synthetically involved, and even subtle structural changes to the parent drug molecule can significantly alter the potency and selectivity profile. ${ }^{3}$ The use of a broadspectrum probe, on the other hand, offers a nearly universal platform of competitive activity-based protein profiling (ABPP). ${ }^{2,4}$ Such a platform can readily measure the proteome-wide action of underivatized covalent drugs, as well as other reactive molecules like environmental toxins and reactive metabolites from the human microbiota. ${ }^{5-8}$ The adaptability of the platform relies on the reactivity coverage of the probe. The broader the coverage, the higher the adaptability.

A widely-adaptable platform of competitive ABPP requires a versatile probe containing a warhead of broad reactivity. It is challenging to design a warhead that covers the diverse reactivity space of a proteome. Individual proteins differ in molecular composition and structure. Their reactions with small molecules are distinct from site to site, domain to domain, and protein to protein. The diversity of these reactions further increases due to the large number and abundance range of proteins in a proteome. Existing probe warheads are not ideal for nearly universal platforms of competitive ABPP. These warheads typically react with one or two types of amino acid residues, therefore selected enzyme classes. Their reactivity depends on either a specific reaction mechanism or a single electrophilic site. ${ }^{8-14}$

We propose a novel use of the $\alpha$-methylene- $\beta$-lactone (MeLac, Scheme 1) moiety as a warhead for covalent probes and inhibitors. MeLac couples an acrylate type of Michael acceptor ${ }^{15-16}$ with a 
$\beta$-lactone. ${ }^{17-19}$ Consequently, this new warhead is prone to reactions with nucleophilic thiol (Cys), hydroxyl (Ser, Thr, and Tyr), and amino (Lys) groups on the protein. Also, MeLac can provide separate sites for regioselective reactions with spatially-arranged protein nucleophiles. Thus, the reactivity of the small and rigid MeLac is expected to be broad. We reason that a MeLac probe would make a competent measurement probe for building a widely-adaptable platform of competitive ABBP.

Using MeLac-alkyne probe 1 (Text S1, Fig. S1), ${ }^{20-21}$ the multi-reactivity of MeLac was investigated (MeLac as the warhead and the alkyne as the reporter). The reactions of proteins with 1 and a reporterless inhibitor 2 in live cells were examined by gel-based ABPP (Fig. 1A, Fig. S2) and characterized, at both protein and peptide/residue resolution, using mass spectrometry (MS)-based proteomics profiling. New methods for the confident identification of protein adducts were developed. A platform of competitive ABBP was built using 1 as an in-live-cell probe. The MeLac-based platform was used for analyzing protein reactions with Orlistat as a model inhibitor for reactive molecules. Detailed experimental conditions were described in Text $\mathbf{S 2}$.

Characterization of a protein reaction with the MeLac probe 1 ultimately requires localizing the reaction site on the protein at a residue resolution. Site localization can be challenging. Gas-phase fragmentation of 1-modified peptides, resulting from trypsinizing reacted proteins, does not always produce the needed sequence ions. These ions are essential evidence to localizing the reaction site at a specific residue. When the site localization is ambiguous, the reaction site is only assignable to the modified peptide (or its fragment) on the corresponding protein. Thus, the protein reaction is characterized at a moderately reduced resolution of peptide level. However, not all reactions can be characterized based on modified peptides. In these cases, the reactions have to be characterized at a heavily reduced resolution of protein level. There are two reasons. Some 1-reacted proteins may not produce modified peptides that are suitable for bottom-up proteomics, the method-of-choice for chemical proteomics. Not all precursor ions of suitable peptides are sampled for MS/MS analysis in proteomics profil- 
ing. With decreasing resolution from residue to protein level, tightened constraints must be set to ensure confidence in reporting reactions of proteins with 1.

Characterization of protein reactions with 1 confronts a primary challenge when reaction sites cannot be assigned to specific residues or peptides. Without the detection of 1-modified peptides, assignment of a 1-protein reaction depends solely on the identification of reacted proteins in enriched preparations. However, these preparations always contain unreacted, background proteins.

We developed a novel method of tagging triplication to differentiate 1-reacted proteins with high confidence from a pool of 1,955 proteins identified by proteomics profiling (Table A1). In each of three separate preparations, a different (desthio)biotin tag was used for avidin-based affinity enrichment of reacted proteins from one lysate sample of 1-reacted HT-29 cells. Our rationale was that the tagged proteins underwent different releasing conditions to recover 1-reacted proteins and thus background proteins in the preparations obtained would be significantly different. When a protein was identified in more than one enrichment preparation, the confidence of it being true 1-reacted protein was increased. Specifically, the 1-reacted proteins were tagged, using copper-catalyzed azide-alkyne cycloaddition (CuAAC), with desthiobiotin azide (Des, Scheme S1), Dde biotin picolyl azide (Dde, Scheme S2) azide or diazo biotin azide (Dia, Scheme S3). Tagged proteins were captured by immobilized NeutrAvidin and then released using different elution conditions for preparing tryptic peptides.

Subsequently, a unique three-tier system was set based on the number of reproduced identifications of a particular protein in the differentially-tagged preparations from a common lysate sample. The lysate was made from HT-29 cells upon in-live-cell reactions with 1. Proteomics profiling identified a total of 1,955 proteins. They were categorized as Tier I, II, or III (white, light grey, and dark grey, respectively; Fig. 1B) with decreasing confidence in being true 1-reacted proteins, accordingly. Proteins in Tier I were identified in all the three tagged samples; Tier II in two; Tier III for only one. Analogous to the minimum requirement of 2 unique peptides for identifying a precursor protein in proteome samples, we assigned Tier I and II proteins as confident identifications; Tier III proteins needed further investiga- 
tion. Indeed, profiling of 1-modified peptides supported that 101 out of 667 proteins in Tier III reacted with 1 (Fig. 1C). Overall, proteomics profiling of enriched 1-reacted proteins supported the broad reactivity of the MeLac warhead with proteins in the proteome, as observed in gel-based ABPP (Fig. 1A) but offered limited information for understanding protein reactions with MeLac.

Proteomics profiling of enriched 1-modified peptides confirmed the broad reactivity of MeLac. A total of 778 modified peptides with 837 modification sites were identified by MaxQuant, ${ }^{22}$ resulting in 634 proteins (Fig. 1D, Table A2). The Andromeda ${ }^{23}$ peptide-spectrum match (PSM) scoring algorithm of MaxQuant was set to filter PSM hits with a minimum score cutoff of 40 and $1 \%$ false discovery rate threshold for both peptide and protein identification. Consequently, for most peptides, alternative PSM assignments with lower scores were discarded. A total of 127 peptides had ambiguous assignments of modification sites; reactions could only be assigned to fragments of modified tryptic peptides. More than 600 peptides had localized modification sites. Moreover, 1-modified peptides originated from functionally diverse proteins, covering enzymatic reactions of all seven general types; these proteins were modified at either catalytic or non-catalytic residues (Table S1, Fig. S3, Fig. S4). Interestingly, in comparison with protein identification from samples from protein-level enrichment, a significant number of proteins were identified only from samples of enriched 1-modified peptides. This observation implied that modified peptide in the enriched peptide samples were further concentrated and the samples were less complex in the sample matrix.

Identification of 1-modified peptides had its own challenge in confidence, differing from the confidence challenge for enriched samples of 1-reacted proteins. The identification of the modified peptides depended only on a single amino acid sequence. Higher-energy collisional dissociation of peptide ions used in this work often generated incomplete sequence ions, preventing de novo sequencing of the peptides. Therefore, we imposed a stringent constraint for verifying MS/MS spectra that could be used for the identification of 1-modified peptides. This constraint was particularly important, because a relatively low PSM score of 40 was set for the database search and identification of modified peptides. 
Verification of a spectral match required the presence of two signature ions from Des as the modification-specific fragment ions (labeled in spectra as $f_{1}$ and $f_{2}$, Fig. 1E and Scheme S4); specificity of these two fragment ions was high, enabling verification of identified 1-modified peptides. Des was introduced to the modified peptide via CuAAC attachment of the Des tag for enriching modified peptides.

MS/MS spectra of 1-modified peptides provided direct and detailed information for characterizing 1-protein reactions. MS/MS spectra with unambiguously localized sites for modification were used. Modification of peptides by 1 was attributable to both Michael addition $\left(\mathrm{Ad}_{\mathrm{M}}\right)$ and acyl addition of MeLac by Cys, Ser, Thr, Tyr, and Lys (Scheme S5, Table A2). Although uncommon and thermodynamically unfavorable based on computation (Text S3, Fig. S5), AdM modifications of Lys and Thr have been reported for proteins. ${ }^{24-25}$ Possible nucleophilic substitutions of the lactone ring of MeLac (Scheme 1), whose reaction products would have degenerate masses as those from acyl addition, were not considered in this work. The modified peptides had three different incremental masses. A global view of MeLac modifications of peptides was apparent in a DeltaMass plot based on unrestricted $\operatorname{search}^{26}$ (Fig. 2A). Upon CuAAC attachment of the Des tag, the modification of a residue by 1 gave a total mass shift of $550.3115 \mathrm{Da}$. Depending on the reaction site on MeLac, further modifications by 1 on reacted peptides happened during sample preparation: hydrolysis of the lactone ring giving an additional increase of 18.0106 $\mathrm{Da}$ (568.3221 Da in total) and quenching of the Michael receptor with 2-mercaptoethanol adding a further increase of 78.0139 Da (628.3254 Da in total) as in Fig. 2B. Modified peptides with a mass increase of 550.3115 Da were attributed to the products of $\mathrm{Ad}_{\mathrm{M}}$ (Scheme S5), but not acyl addition; large excess of 2-mercaptoethanol was used for quenching the Michael acceptor and the thioester bond formed via acyl addition of the $\beta$-lactone ring is labile. ${ }^{27}$ Indeed, few modified peptides could be assigned to be quenched products from the acyl addition of MeLac by Cys.

The cysteinome provides many important targets for covalent drugs. ${ }^{28}$ The majority of 1modified peptides carried a localized site of Cys modification. This observation was not surprising, considering the large numbers of reactive cysteines under physiological conditions. Thus, we compared 
MeLac probe 1 with iodoacetamide (IA)-alkyne, which is routinely used as a broad-spectrum cysteinereactive probe in chemical proteomics. Localized IA reaction sites on 6143 cysteinyl peptides had been reported, among which 758 sites were ligandable. ${ }^{6}$ Although sharing 128 ligandable peptides with the IA-alkyne pool, 224 out of the 653 peptides with 1-modification either catalytic or non-catalytic cysteines were unique to MeLac (Fig. S6). It is important to note that beside the reaction chemistry difference between MeLac and IA, MDA-MB-231 and Ramos cells were used in the IA study, and the IAalkyne probe was used at $100 \mu \mathrm{M} .{ }^{6}$ In comparison, $\mathrm{HT}-29$ cells were used in our work with 1 at concentrations up to $20 \mu \mathrm{M}$. Systematic experiments are needed to compare utilities of probes with the IA and MeLac warheads for measuring reactive cysteines proteome-wide.

A significant advantage of MeLac over IA probes comes from difference in cytotoxicity. IA has high cytotoxicity, ${ }^{29}$ limiting application of IA probes mainly to lysates. ${ }^{30}$ In contrast, MeLac compounds were used with live cells at concentrations up to $100 \mu \mathrm{M}$ for 1 and $300 \mu \mathrm{M}$ for MeLac inhibitor 2 in this work. Cells remained adhered to culture plates under these concentrations for incubation times up to 1 hr. Two important analytical advantages from the live-cell application of a measurement probe are (1) decreased background reactions and (2) enabled analysis of proteases whose activity has to be blocked during cell lysis. Additionally, MeLac-based competitive ABPP for analyzing the action of noncovalent inhibitors in live cells is a unique potential.

We then moved on to test a major designed utility of MeLac probes in competitive ABPP platforms for analyzing proteome-wide reactions of reactive molecules. We chose Orlistat as a model covalent inhibitor, a $\beta$-lactone compound capable of forming covalent adducts with nucleophilic hydroxyl and thiol groups on proteins. Orlistat is an FDA approved drug that targets lipases for weight management. Extensive chemical proteomics analysis has revealed many off-target proteins, ${ }^{3,27}$ which are linked to antitumor activities ${ }^{31}$ as well as organ toxicity ${ }^{32}$ for this over-the-counter drug. Most of the covalent Orlistat-protein adducts are formed via a labile thioester bond. ${ }^{27}$ Confident identification of off-target proteins of Orlistat, especially Orlistat-modified peptide, is challenged by the fact that the thioester 
bond is not stable during sample preparation for proteomics analysis. This is a common problem for $\beta$ lactone based probes. Live-cell competitive ABPP using 1 as the measurement probe provides a solution to this analysis challenge.

Upon Orlistat (1 and $10 \mu \mathrm{M})$ treatment of HT-29 cells, the remaining quantity of unengaged proteins, which then reacted with the MeLac probe 1 at $20 \mu \mathrm{M}$, was compared based on 1-modified peptides. These peptides were quantified using a label-free method. For competitive ABPP platforms using a broad-reactivity probe like 1, direct measurement of the probe-modified peptides depicts reactive molecules reacting with proteins at a peptide-level resolution, as demonstrated in the Orlistate-GSTP1 example below. In contrast, the quantitation of probe-reacted proteins produces indirect, possibly compromised results. When a measurement probe has a broad reactivity, it could react with different regions of a protein. Consequently, fold change (FC) measured based on the relative amount of probereacted protein as a whole is compressed due to possible probe reactions at other sites of the protein that are not competed by the reactive molecule of interest, e.g., Orlistat. Given the ratio compression issue at the protein level of quantitation, a volcano plot of $p$-value against FC was constructed based on 1-modified peptides for analyzing Orlistat reactions with the corresponding proteins. When the modified peptides had $>4$-fold decrease in quantity with $p<0.05$, reactions of 1 with proteins were considered being competed off by Orlistat (Fig. 3). A total of 23 modified peptides (and thus their precursor proteins) qualified the FC- $p$ cutoff in the concentration window of Orlistat between 1 and $10 \mu \mathrm{M}$ (Table S2). It should be noted that peptides not passing the cutoff were not assigned as being not competing, because the dose window of Orlistat was narrow and label-free quantitation that has less precision than isotope-labeling based quantitation was used.

Peptide YISLIYTNYEAGKDDYVK from glutathione S-transferase P1 (P09211, GSTP1) had a 14.6-fold ( $p=0.004)$ decrease in the quantity for the Orlistat reaction at $10 \mu \mathrm{M}$ over $1 \mu \mathrm{M}$ (Fig. 3). This significant decrease measured by 1 strongly indicated that Orlistat occupied in the same region on the protein as where 1 reacted. Intriguingly, multiple MS/MS spectra observed different modification sites of 
1 on this peptide (Fig. S7) and the peptide locates in a region of GSTP1 where different nucleophiles coexist (Insert of Fig. 3).

In summary, by coupling a Michael acceptor with a $\beta$-lactone moiety, a novel warhead, MeLac, for chemical probes has been generated. Broad reactivity of this small and rigid warhead allows it to react with different protein nucleophiles through distinct mechanisms. Multiple reactive sites on MeLac extend the scope of these reactions and provide a potential means of conjugating recognizing moieties for ligand-directed chemistry ${ }^{33}$ in live cells. With its broad reactivity, the MeLac warhead also offers a unique possibility to convert non-covalent inhibitors to measurement probes targeting different enzymes, ${ }^{34}$ as well as to broaden druggable targets by identifying new binding sites. ${ }^{6-7}$ MeLac probes, being useful for live-cell applications, potentially have an immense impact on drug discovery and further expand the analysis of the reactions with proteins that can deactivate during cell lysis. MeLacbased competitive ABPP platforms are highly adaptable and are appropriate for measuring the live-cell action of a wide array of small reactive molecules. 

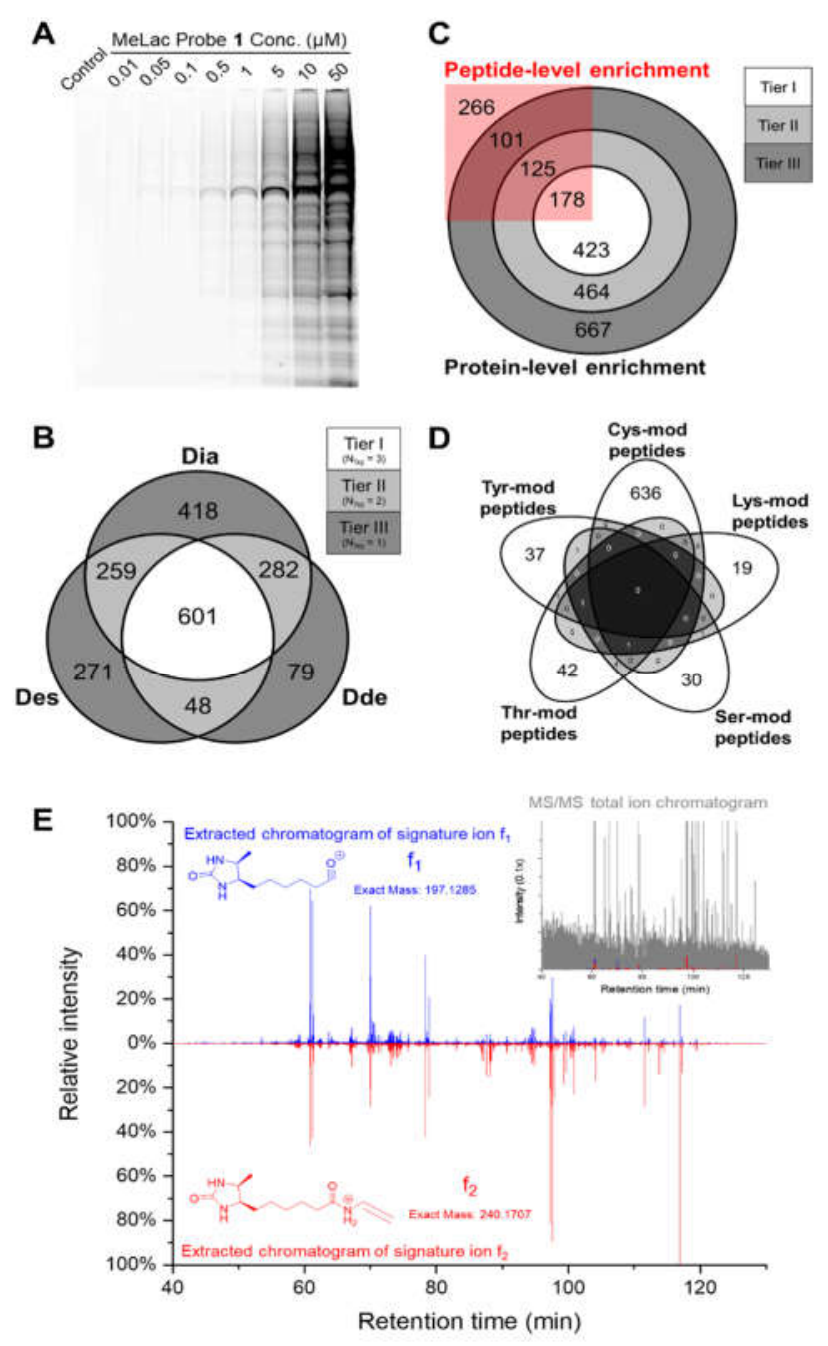

Figure 1. Characterization of protein reactions with $\alpha$-methylene- $\beta$-lactone (MeLac) probe. (A) Gelbased activity-based protein profiling showing broad reactivity of the MeLac probe. (B) Triplicated selective enrichment of probe-reacted proteins resulting in confident identification of probe-reacted proteins at protein resolution. (C) Comparison between protein-level and peptide level identification. (D) Probe-modified peptides identified at a peptide-level resolution. (E) Signature ion-based reduction of data complexity and validation of identification of probe-modified peptide; insert comparing total ion chromatogram of peptide fragment ions and extracted ion chromatograms for highly specific fragment ions of desthiobiotin (f1 and f2). 


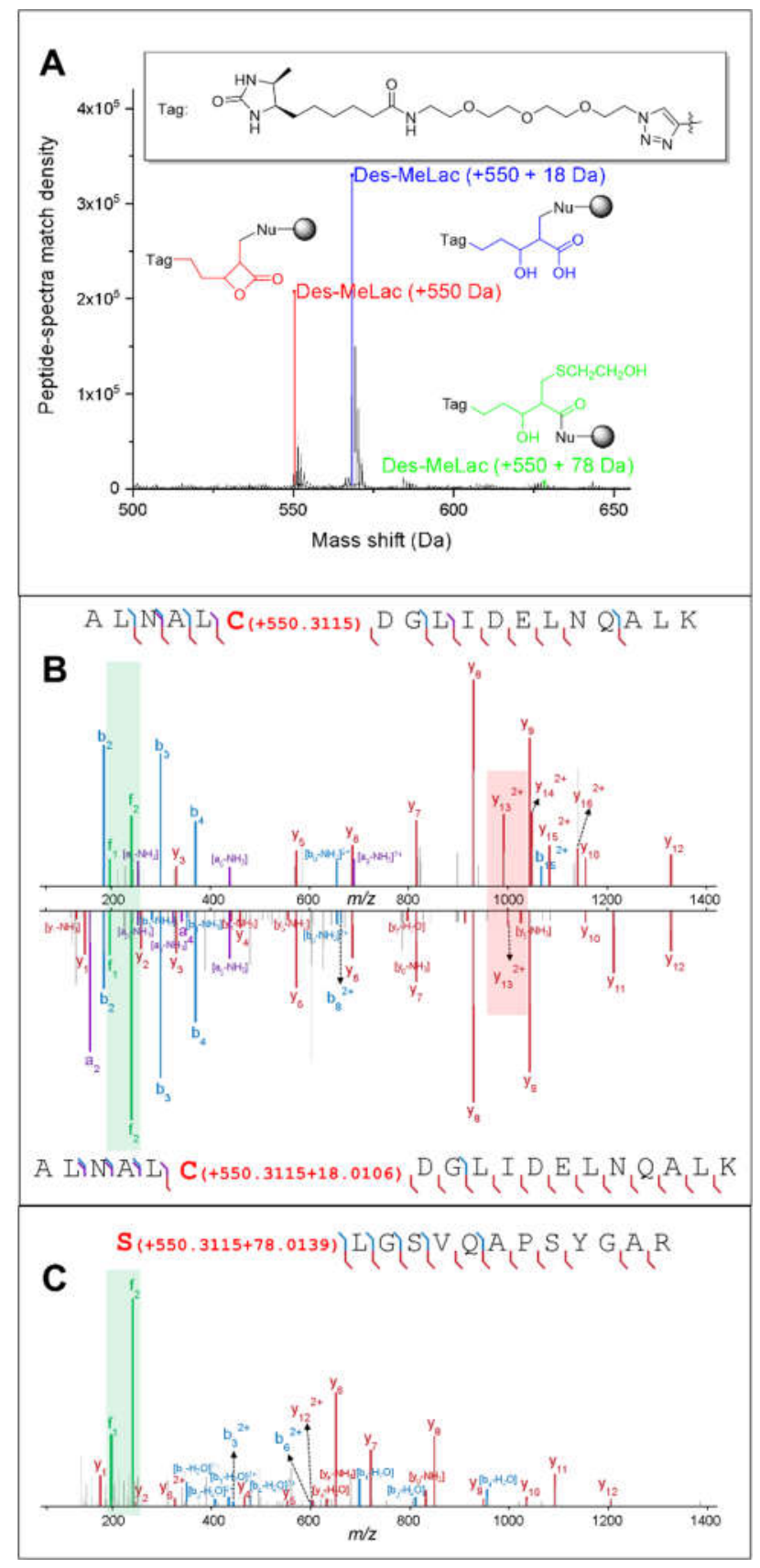

Figure 2. Global and residue-specific illustration of MeLac modifications on peptides. (A) A DeltaMass plot showing global modification of peptides with incremental masses attributable to MeLac reactions. (B) Representative MeLac modifications on peptides at Cys residue. (C) Representative MeLac modification on peptides at Ser residue. 


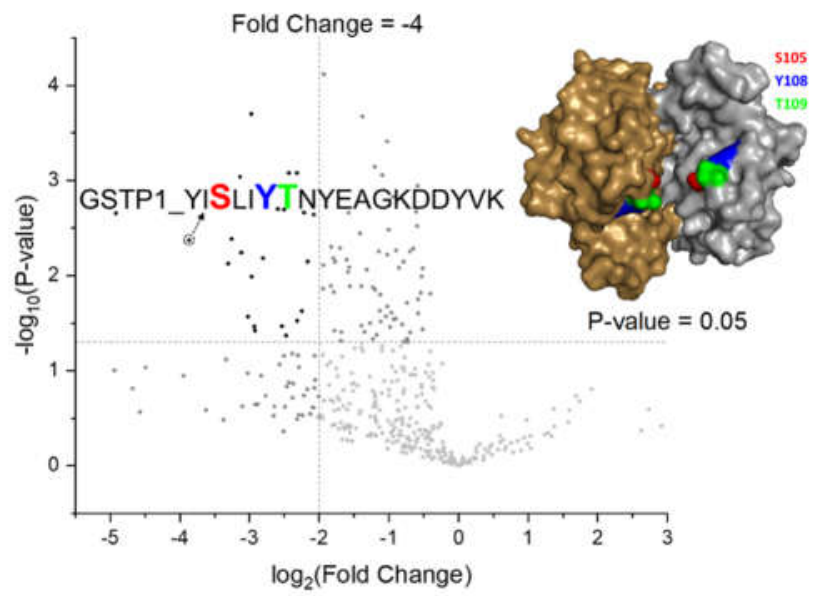

Figure 3. MeLac probe measuring selective protein reactions with Orlistat at a peptide level resolution. The volcano plot shows that Orlistat selectively reacts with 23 proteins at the regions of probe-modified peptides (black dots, upper left; $<0.05$ for $p$-value and $>4$ for fold change). These reactions are detectable by changing the Orlistat concentration from 1 to $10 \mu \mathrm{M}$, using the MeLac measurement probe. A GSTP1 peptide is labeled with its sequence. Structure: GSTP1 dimmer with colored residues bearing the MeLac modification. 
Scheme 1. MeLac warhead (left), MeLac-alkyne probe (1), and alkyl MeLac inhibitor (2).

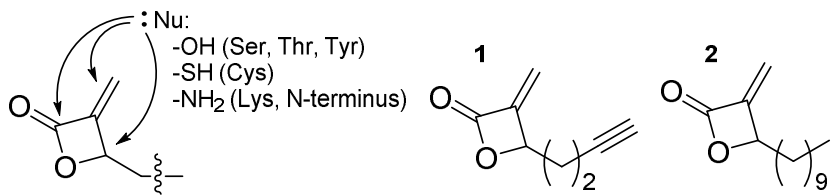




\section{AUTHOR INFORMATION}

\section{Corresponding Authors}

Amy R. Howell and Xudong Yao - Department of Chemistry, University of Connecticut, Storrs, Connecticut 06269, USA

Emails: amy.howell@uconn.edu; x.yao@uconn.edu

\section{Author Contributions}

L.W., B.B and X.Y. designed and implemented chemical proteomics workflows, performed analyses and data interpretation, and drafted the manuscript. L.W. and X.Y. performed restricted and open search of LC-MS/MS data and results visualization. L.W. conducted quantum mechanical computation of model reactions. L.P.R. and A.R.H. synthesized and characterized the $\alpha$-methylene- $\beta$-lactone (MeLac) probe and inhibitor and revised the manuscript. B.D. performed LC-MS/MS analysis of proteome samples, wrote the related experimental, and read the manuscript. A,R.H. designed the synthesis of MeLac compounds. X.Y. perceived the use of the MeLac warhead in covalent probes and inhibitors and designed the project overall. All authors have given approval to the final version of the manuscript.

\section{ACKNOWLEDGMENT}

A generous donation from the Estate of Anthony DeThomas to the Division of Analytical Chemistry of the Department of Chemistry at the University of Connecticut is gratefully appreciated. The Vermont Genetics Network Proteomics Facility that produced LC-MS/MS data for selected samples is supported through NIH Grant P20GM103449 from the INBRE Program of the National Institute of General Medical Sciences.

\section{ABBREVIATIONS}


MeLac, $\alpha$-Methylene- $\beta$-lactone; ABPP, activity-based protein profiling; MS, mass spectrometry; CuAAC, copper-catalyzed azide-alkyne cycloaddition; Des, desthiobiotin azide; Dde, Dde biotin picolyl azide; Dia, diazo biotin azide; PSM, peptide-spectrum match; AdM, Michael addition; IA, iodoacrylamidealkyne; GSTP1, glutathione S-transferase P1. 


\section{REFERENCES}

1. Baillie, T. A., Targeted Covalent Inhibitors for Drug Design. Angew Chem Int Ed Engl 2016, 55 (43), 13408-13421.

2. Moellering, R. E.; Cravatt, B. F., How chemoproteomics can enable drug discovery and development. Chem Biol 2012, 19 (1), 11-22.

3. Yang, P. Y.; Liu, K.; Zhang, C.; Chen, G. Y.; Shen, Y.; Ngai, M. H.; Lear, M. J.; Yao, S. Q., Chemical modification and organelle-specific localization of orlistat-like natural-product-based probes. Chem Asian J 2011, 6 (10), 2762-75.

4. Lanning, B. R.; Whitby, L. R.; Dix, M. M.; Douhan, J.; Gilbert, A. M.; Hett, E. C.; Johnson, T. O.; Joslyn, C.; Kath, J. C.; Niessen, S.; Roberts, L. R.; Schnute, M. E.; Wang, C.; Hulce, J. J.; Wei, B.; Whiteley, L. O.; Hayward, M. M.; Cravatt, B. F., A road map to evaluate the proteome-wide selectivity of covalent kinase inhibitors. Nat Chem Biol 2014, 10 (9), 760-767.

5. Tuley, A.; Fast, W., The Taxonomy of Covalent Inhibitors. Biochemistry 2018, 57 (24), 33263337.

6. Backus, K. M.; Correia, B. E.; Lum, K. M.; Forli, S.; Horning, B. D.; Gonzalez-Paez, G. E.; Chatterjee, S.; Lanning, B. R.; Teijaro, J. R.; Olson, A. J.; Wolan, D. W.; Cravatt, B. F., Proteome-wide covalent ligand discovery in native biological systems. Nature 2016, 534 (7608), 570-4.

7. Hacker, S. M.; Backus, K. M.; Lazear, M. R.; Forli, S.; Correia, B. E.; Cravatt, B. F., Global profiling of lysine reactivity and ligandability in the human proteome. Nat Chem 2017, 9 (12), 11811190.

8. Cuesta, A.; Taunton, J., Lysine-Targeted Inhibitors and Chemoproteomic Probes. Annu Rev Biochem 2019, 88, 365-381. 
9. Gehringer, M.; Laufer, S. A., Emerging and Re-Emerging Warheads for Targeted Covalent Inhibitors: Applications in Medicinal Chemistry and Chemical Biology. J Med Chem 2019, 62 (12), 5673-5724.

10. Ray, S.; Murkin, A. S., New Electrophiles and Strategies for Mechanism-Based and Targeted Covalent Inhibitor Design. Biochemistry 2019, 58 (52), 5234-5244.

11. Abranyi-Balogh, P.; Petri, L.; Imre, T.; Szijj, P.; Scarpino, A.; Hrast, M.; Mitrovic, A.; Fonovic, U. P.; Nemeth, K.; Barreteau, H.; Roper, D. I.; Horvati, K.; Ferenczy, G. G.; Kos, J.; Ilas, J.; Gobec, S.; Keseru, G. M., A road map for prioritizing warheads for cysteine targeting covalent inhibitors. Eur J Med Chem 2018, 160 (DOI: 10.1016/j.ejmech.2018.10.010), 94-107.

12. Jones, L. H., Reactive Chemical Probes: Beyond the Kinase Cysteinome. Angew Chem Int Ed Engl 2018, 57 (30), 9220-9223.

13. Mukherjee, H.; Debreczeni, J.; Breed, J.; Tentarelli, S.; Aquila, B.; Dowling, J. E.; Whitty, A.; Grimster, N. P., A study of the reactivity of $\mathrm{S}((\mathrm{VI}))$-F containing warheads with nucleophilic amino-acid side chains under physiological conditions. Org Biomol Chem 2017, 15 (45), 9685-9695.

14. Ma, N.; Hu, J.; Zhang, Z. M.; Liu, W.; Huang, M.; Fan, Y.; Yin, X.; Wang, J.; Ding, K.; Ye, W.; Li, Z., 2H-Azirine-Based Reagents for Chemoselective Bioconjugation at Carboxyl Residues Inside Live Cells. J Am Chem Soc 2020.

15. Kathman, S. G.; Xu, Z.; Statsyuk, A. V., A fragment-based method to discover irreversible covalent inhibitors of cysteine proteases. J Med Chem 2014, 57 (11), 4969-74.

16. Previti, S.; Ettari, R.; Cosconati, S.; Amendola, G.; Chouchene, K.; Wagner, A.; Hellmich, U. A.; Ulrich, K.; Krauth-Siegel, R. L.; Wich, P. R.; Schmid, I.; Schirmeister, T.; Gut, J.; Rosenthal, P. J.; Grasso, S.; Zappala, M., Development of Novel Peptide-Based Michael Acceptors Targeting Rhodesain 
and Falcipain-2 for the Treatment of Neglected Tropical Diseases (NTDs). J Med Chem 2017, 60 (16), 6911-6923.

17. Böttcher, T.; Sieber, S. A., $\beta$-Lactams and $\beta$-lactones as activity-based probes in chemical biology. MedChemComm 2012, 3 (4), 408-408.

18. Kamat, S. S.; Camara, K.; Parsons, W. H.; Chen, D. H.; Dix, M. M.; Bird, T. D.; Howell, A. R.; Cravatt, B. F., Immunomodulatory lysophosphatidylserines are regulated by ABHD16A and ABHD12 interplay. Nat Chem Biol 2015, 11 (2), 164-71.

19. Li, S.; Diego-Limpin, P. A.; Bajrami, B.; Keshipeddy, S.; Lam, Y. W.; Deng, B.; Farrokhi, V.; McShane, A. J.; Nemati, R.; Howell, A. R.; Yao, X., Scaling Proteome-Wide Reactions of Activity-Based Probes. Anal Chem 2017, 89 (12), 6295-6299.

20. Camara, K.; Kamat, S. S.; Lasota, C. C.; Cravatt, B. F.; Howell, A. R., Combining crossmetathesis and activity-based protein profiling: new beta-lactone motifs for targeting serine hydrolases. Bioorg Med Chem Lett 2015, 25 (2), 317-21.

21. Malapit, C. A.; Caldwell, D. R.; Sassu, N.; Milbin, S.; Howell, A. R., Pd-Catalyzed Acyl C-O Bond Activation for Selective Ring-Opening of alpha-Methylene-beta-lactones with Amines. Org Lett 2017, 19 (8), 1966-1969.

22. Tyanova, S.; Temu, T.; Cox, J., The MaxQuant computational platform for mass spectrometrybased shotgun proteomics. Nat Protoc 2016, 11 (12), 2301-2319.

23. Cox, J.; Neuhauser, N.; Michalski, A.; Scheltema, R. A.; Olsen, J. V.; Mann, M., Andromeda: a peptide search engine integrated into the MaxQuant environment. J Proteome Res 2011, 10 (4), 1794805. 
24. Pettinger, J.; Le Bihan, Y. V.; Widya, M.; van Montfort, R. L.; Jones, K.; Cheeseman, M. D., An Irreversible Inhibitor of HSP72 that Unexpectedly Targets Lysine-56. Angew Chem Int Ed Engl 2017, 56 (13), 3536-3540.

25. Groll, M.; Schellenberg, B.; Bachmann, A. S.; Archer, C. R.; Huber, R.; Powell, T. K.; Lindow, S.; Kaiser, M.; Dudler, R., A plant pathogen virulence factor inhibits the eukaryotic proteasome by a novel mechanism. Nature 2008, 452 (7188), 755-8.

26. Avtonomov, D. M.; Kong, A.; Nesvizhskii, A. I., DeltaMass: Automated Detection and Visualization of Mass Shifts in Proteomic Open-Search Results. J Proteome Res 2019, 18 (2), 715720.

27. Yang, P. Y.; Liu, K.; Ngai, M. H.; Lear, M. J.; Wenk, M. R.; Yao, S. Q., Activity-based proteome profiling of potential cellular targets of Orlistat--an FDA-approved drug with anti-tumor activities. J Am Chem Soc 2010, 132 (2), 656-66.

28. Maurais, A. J.; Weerapana, E., Reactive-cysteine profiling for drug discovery. Curr Opin Chem Biol 2019, 50, 29-36.

29. Lin, D.; Saleh, S.; Liebler, D. C., Reversibility of covalent electrophile-protein adducts and chemical toxicity. Chem Res Toxicol 2008, 21 (12), 2361-9.

30. Abo, M.; Weerapana, E., A Caged Electrophilic Probe for Global Analysis of Cysteine Reactivity in Living Cells. J Am Chem Soc 2015, 137 (22), 7087-90.

31. Kridel, S. J.; Axelrod, F.; Rozenkrantz, N.; Smith, J. W., Orlistat is a novel inhibitor of fatty acid synthase with antitumor activity. Cancer Res 2004, 64 (6), 2070-5. 
32. Xiao, D.; Shi, D.; Yang, D.; Barthel, B.; Koch, T. H.; Yan, B., Carboxylesterase-2 is a highly sensitive target of the antiobesity agent orlistat with profound implications in the activation of anticancer prodrugs. Biochem Pharmacol 2013, 85 (3), 439-47.

33. Tamura, T.; Ueda, T.; Goto, T.; Tsukidate, T.; Shapira, Y.; Nishikawa, Y.; Fujisawa, A.; Hamachi, I., Rapid labelling and covalent inhibition of intracellular native proteins using ligand-directed N-acyl-Nalkyl sulfonamide. Nat Commun 2018, 9 (1), 1870.

34. Zhao, Q.; Ouyang, X.; Wan, X.; Gajiwala, K. S.; Kath, J. C.; Jones, L. H.; Burlingame, A. L.; Taunton, J., Broad-Spectrum Kinase Profiling in Live Cells with Lysine-Targeted Sulfonyl Fluoride Probes. J Am Chem Soc 2017, 139 (2), 680-685. 


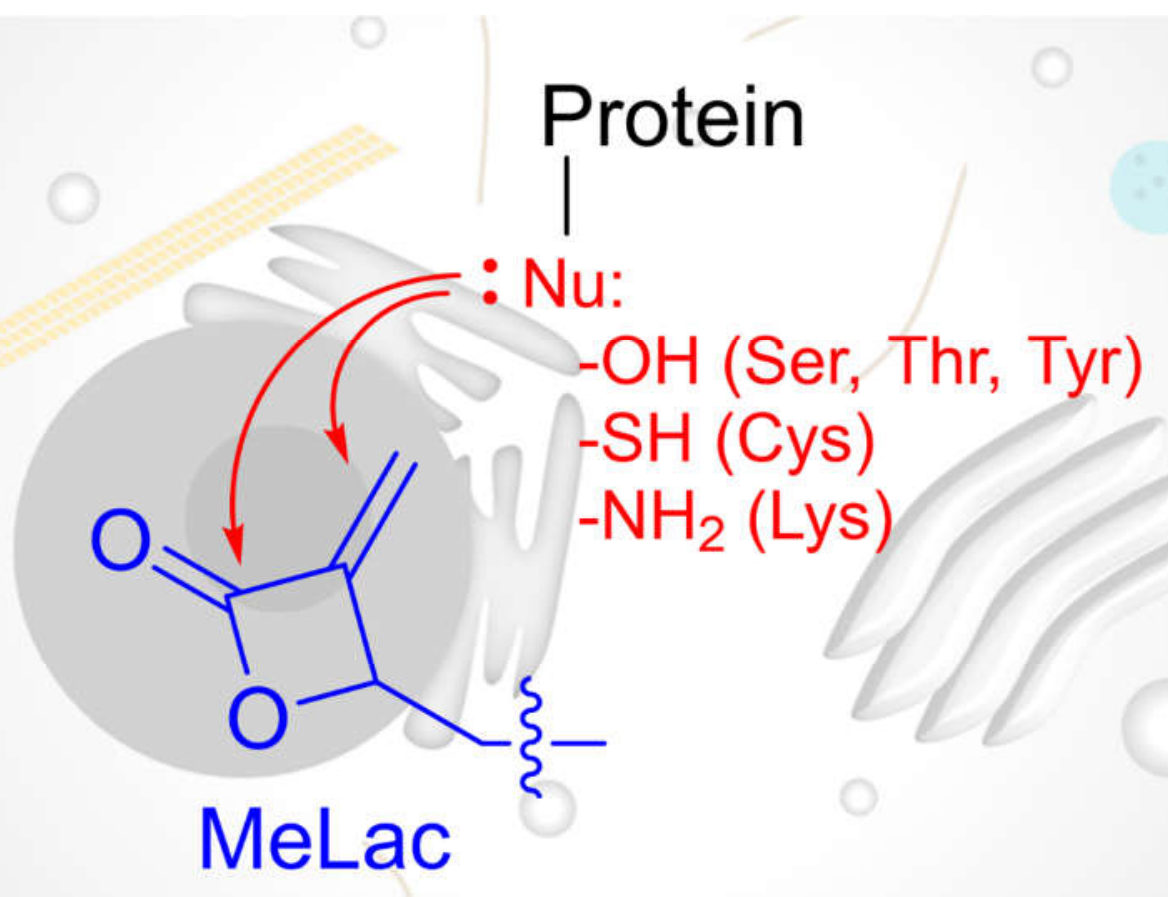

